\title{
ABCD Matrices as Similarity Transformations of Wigner Matrices and Periodic Systems in Optics
}

\author{
S. Başkal 1 \\ Department of Physics, Middle East Technical University, 06531 Ankara, \\ Turkey \\ Y. S. Kim 2 \\ Department of Physics, University of Maryland, \\ College Park, Maryland 20742
}

\begin{abstract}
The beam transfer matrix, often called the $A B C D$ matrix, is a two-by-two matrix with unit determinant, and with three independent parameters. It is noted that this matrix cannot always be diagonalized. It can however be brought by rotation to a matrix with equal diagonal elements. This equi-diagonal matrix can then be squeezetransformed to a rotation, to a squeeze, or to one of the two shear matrices. It is noted that these one-parameter matrices constitute the basic elements of the Wigner's little group for space-time symmetries of elementary particles. Thus every $A B C D$ matrix can be written as a similarity transformation of one of the Wigner matrices, while the transformation matrix is a rotation preceded by a squeeze. This mathematical property enables us to compute scattering processes in periodic systems. Laser cavities and multilayer optics are discussed in detail. For both cases, it is shown possible to write the one-cycle transfer matrix as a similarity transformation of one of the Wigner matrices. It is thus possible to calculate the $A B C D$ matrix for an arbitrary number of cycles.
\end{abstract}

\footnotetext{
${ }^{1}$ electronic address: baskal@newton.physics.metu.edu.tr

${ }^{2}$ electronic address: yskim@physics.umd.edu
} 


\section{Introduction}

Without too much exaggeration, modern physics consists of harmonic oscillators and two-by-two matrices. Ray optics is mostly the physics of two-by-two matrices. The beam transfer matrix, usually called the $A B C D$ matrix, is a two-by-two matrix with unit determinant and with three independent parameters.

In mathematics, the group of matrices with this property is called the two-dimensional symplectic group or $S p(2)$. Indeed, the $S p(2)$ group has been playing a pivotal role both in classical and quantum optics. It is the basic language for lens optics [2, 3], multilayer optics [4, 5], laser cavities [6], as well as squeezed states of light in quantum optics [7, 8].

This group is locally isomorphic to the group of Lorentz transformations applicable to one time dimension and two space dimensions. This Lorentz group consists of three-by-three matrices. Since the algebra of two-by-two matrices is much simpler than those of three-by-three, the $S p(2)$ group provides a very convenient computational tool for Lorentz transformations in physics 9].

While our understanding of the Pauli matrices and the rotation group is thorough, we are not yet completely familiar with the matrices of the $S p(2)$ group [10, 11]. Do we know how to diagonalize the $A B C D$ matrix? We shall point out in this paper that, unlike the case of the rotation matrices, it is not always possible to digonalize the $A B C D$ matrix by a similarity transformation. We shall show however that every $A B C D$ matrix can be rotated to a matrix with equal diagonal elements.

We shall then show that this equi-diagonal matrix can be squeezed to one of the four one-parameter matrices consisting rotation, squeeze, and two shear matrices. They take the form

$$
\begin{aligned}
& \left(\begin{array}{cc}
\cos \theta^{*} & -\sin \theta^{*} \\
\sin \theta^{*} & \cos \theta^{*}
\end{array}\right), \quad\left(\begin{array}{ll}
\cosh \lambda^{*} & \sinh \lambda^{*} \\
\sinh \lambda^{*} & \cosh \lambda^{*}
\end{array}\right), \\
& \left(\begin{array}{cc}
1 & -\gamma^{*} \\
0 & 1
\end{array}\right), \quad\left(\begin{array}{cc}
1 & 0 \\
\gamma^{*} & 1
\end{array}\right) .
\end{aligned}
$$

One of the two triangular matrices in the above expression can be obtained from the other by rotation, but it is more convenient to use both of them.

It should be noted that these four matrices form the four basic matrices of Wigner's little group for space-time symmetries for elementary particles [12, 
13. We shall therefore call them Wigner matrices, and use $W$ collectively for these four matrices.

These matrices have equal diagonal elements, but not all of them are diagonalizable. The first matrix is a rotation matrix, whose properties are well known. However, its eigenvalues are complex, and therefore it cannot be diagonalized if all the matrices in the group are to be real. The second matrix is symmetric and can be diagonalized by a rotation. The third and fourth matrices are triangular and cannot be diagonalized.

On the other hand, these matrices share one convenient feature with the diagonal matrix. Namely, for the first matrix,

$$
\begin{array}{r}
\left(\begin{array}{cc}
\cos \theta_{1} & -\sin \theta_{1} \\
\sin \theta_{1} & \cos \theta_{1}
\end{array}\right)\left(\begin{array}{cc}
\cos \theta_{2} & -\sin \theta_{2} \\
\sin \theta_{2} & \cos \theta_{2}
\end{array}\right) \\
=\left(\begin{array}{cc}
\cos \left(\theta_{1}+\theta_{2}\right) & -\sin \left(\theta_{1}+\theta_{2}\right) \\
\sin \left(\theta_{1}+\theta_{2}\right) & \cos \left(\theta_{1}+\theta_{2}\right)
\end{array}\right),
\end{array}
$$

and thus

$$
\left(\begin{array}{cc}
\cos \theta & -\sin \theta \\
\sin \theta & \cos \theta
\end{array}\right)^{N}=\left(\begin{array}{cc}
\cos (N \theta) & -\sin (N \theta) \\
\sin (N \theta) & \cos (N \theta)
\end{array}\right),
$$

The multiplication of two matrices results in the addition of parameters. This was called "slide-rule" property in reference [5], but it is more appropriate to call it "logarithmic property." The remaining three matrices in equation (11) have this logarithmic property.

We shall show in this paper that the $A B C D$ matrix can be brought to one of the Wigner matrices by a similarity transformation, and that the similarity transformation is a rotation followed by a squeeze.

This mathematical result can be useful in calculating the $A B C D$ matrix for periodic systems. In this paper, we study laser cavities and multilayer optics in detail. In both cases, we reduce the multi-cycles system into one cycle. Both subjects have been extensively discussed in the literature.

Recently, the present authors used the method of Wigner's little group to calculate the $A B C D$ matrix for laser cavities [2]. However, their cycle had to start from the midway between the two mirrors. In this paper, our cycle will start at an arbitrary point.

In 2003, Georgieva and Kim used a method based on the Lorentz group to study multilayer optics [5]. These authors also end up with the inconvenience of starting their cycle from the midway in one of the layers. We eliminate this inconvenience. 
In Sec. 2, the eigenvalues of the $A B C D$ matrix is discussed in detail. They can be real, complex, and one. Not all of them can be brought to a diagonal form, especially if we restrict the matrices to be real. It is noted however that every $A B C D$ matrix can be brought to a form with equal diagonal elements by a rotation. In Sec. 3, we construct a similarity transformation which will bring the $A B C D$ matrix into one of the four Wigner matrices. It is shown that this matrix is a rotation matrix followed by a squeeze matrix. It is also shown that these four different Wigner matrices can be combined into one analytical expression.

The present formalism is applicable to one-dimensional periodic systems. In Sec. 4, we study laser cavities in detail. In Sec. 5, we show how useful Wigner matrices are in computing the scattering matrix for multilayer optics.

\section{Eigenvalues of the ABCD matrix}

Let us start with the two-by-two matrix

$$
\left(\begin{array}{ll}
A & B \\
C & D
\end{array}\right)
$$

where the elements $A, B, C, D$ are real. The determinant of this matrix is one and thus $(A D-B C)=1$.

The eigenvalue equation for this two-by-two matrix is only a quadratic equation, and it is always soluble. The eigenvalue equation becomes

$$
E^{2}-(A+D) E+A D-B C=0
$$

and the eigenvalues are

$$
E_{ \pm}=\frac{1}{2}\left(A+D \pm \sqrt{(A-D)^{2}+4 B C}\right) .
$$

If the quantity inside the square root sign is negative and $(A+D) / 2$ is smaller than one, then the eigenvalues become

$$
E_{ \pm}=\exp \left( \pm i \theta^{*}\right)
$$

with

$$
\cos \theta^{*}=\frac{A+D}{2}
$$


If the quantity inside the square root sign is positive, and $(A+D) / 2$ is greater than one. The eigenvalues are

$$
E_{ \pm}=\exp \left( \pm \lambda^{*}\right)
$$

with

$$
\cosh \lambda^{*}=\frac{A+D}{2} .
$$

If the quantity inside the square root sign vanishes,

$$
E_{ \pm}=1
$$

These are all well known, and the purpose of this section is not to repeat these trivial procedures.

Although we are used to diagonal matrices with eigenvalues as diagonal elements, it is very cumbersome to deal with the above three separate cases. In order to find a common ground, we propose to use the matrix of the form with equal diagonal elements:

$$
\left(\begin{array}{cc}
J & F \\
G & J
\end{array}\right)
$$

and propose to bring the $A B C D$ matrix of equation (4) to this form by a similarity transformation. Since the trace is preserved under similarity transformations,

$$
J=\frac{A+D}{2}
$$

Since the determinant of the $A B C D$ matrix is one,

$$
J^{2}-F G=1
$$

In this paper, we started with the $A B C D$ matrix with three independent parameters. Because of the condition on the diagonal elements, the $J F G J$ matrix has two independent parameters. If the off diagonal elements $F$ and $G$ have opposite signs, it can be written as

$$
\left(\begin{array}{cc}
\cos \theta^{*} & -e^{\eta} \sin \theta^{*} \\
e^{-\eta} \sin \theta^{*} & \cos \theta^{*}
\end{array}\right)
$$

If $F$ and $G$ have the same sign, it can take the form

$$
\left(\begin{array}{cc}
\cosh \lambda^{*} & e^{\eta} \sinh \lambda^{*} \\
e^{-\eta} \sinh \lambda^{*} & \cos \lambda^{*}
\end{array}\right) .
$$


If $F$ or $G$ vanishes, we can write the $J F G J$ matrix as

$$
\left(\begin{array}{cc}
1 & e^{\eta} \gamma^{*} \\
0 & 1
\end{array}\right), \text { or }\left(\begin{array}{cc}
1 & 0 \\
e^{-\eta} \gamma^{*} & 1
\end{array}\right) \text {. }
$$

Let us go back to equation (11), the above matrices are similarity transformations of the Wigner matrices with the transformation matrix

$$
\left(\begin{array}{cc}
e^{\eta / 2} & 0 \\
0 & e^{-\eta / 2}
\end{array}\right),
$$

which expands one axis and contracts the other in the two-dimensional space. This is a squeeze transformation. In general, symmetric two-by-two matrices perform squeeze transformations. Indeed, the matrices in equations (15) to (17) are "squeezed" Wigner matrices.

\section{Similarity Transformations}

In order to find a similarity transformation which will bring the $A B C D$ matrix of equation (4) to the equi-diagonal matrix of equation (12), let us try a rotation

$$
\left(\begin{array}{cc}
\cos (\delta / 2) & \sin (\delta / 2) \\
-\sin (\delta / 2) & \cos (\delta / 2)
\end{array}\right)\left(\begin{array}{cc}
A & B \\
C & D
\end{array}\right)\left(\begin{array}{cc}
\cos (\delta / 2) & -\sin (\delta / 2) \\
\sin (\delta / 2) & \cos (\delta / 2)
\end{array}\right) .
$$

We can calculate the angle $\delta$ first. The result is

$$
\tan \delta=\frac{D-A}{B+C}
$$

Using this angle, we can then compute $F$ and $G$. They are

$$
\begin{aligned}
& F=\frac{1}{2}\left(B-C+\sqrt{(A-D)^{2}+(B+C)^{2}}\right), \\
& G=\frac{1}{2}\left(C-B+\sqrt{(A-D)^{2}+(B+C)^{2}}\right) .
\end{aligned}
$$

If $J$ is smaller than one, we can write $J=\cos \theta^{*}$, and use equation (15) for the JFGJ matrix, with

$$
\begin{aligned}
& \cos \theta^{*}=\frac{A+D}{2} \\
& e^{2 \eta}=\frac{C-B-\sqrt{(A-D)^{2}+(B+C)^{2}}}{C-B+\sqrt{(A-D)^{2}+(B+C)^{2}}},
\end{aligned}
$$


and the Wigner matrix should take the form

$$
\left(\begin{array}{cc}
\cos \theta^{*} & -\sin \theta^{*} \\
\sin \theta^{*} & \cos \theta^{*}
\end{array}\right)
$$

which is one of the four matrices given in equation (1).

If $J$ is greater than 1 , we should use equation (16), with

$$
\begin{aligned}
& \cosh \lambda^{*}=\frac{A+D}{2} \\
& e^{2 \eta}=\frac{B-C+\sqrt{(A-D)^{2}+(B+C)^{2}}}{C-B+\sqrt{(A-D)^{2}+(B+C)^{2}}} .
\end{aligned}
$$

The Wigner matrix in this case is

$$
\left(\begin{array}{cc}
\cosh \lambda^{*} & \sin \lambda^{*} \\
\cosh \lambda^{*} & \sinh \lambda^{*}
\end{array}\right)
$$

which is also given in equation (1).

If $J=1$, the diagonal elements are one, and the off-diagonal element $F$ or $G$ has to vanish. As a consequence, the $A B C D$ matrix becomes

$$
\left(\begin{array}{cc}
1 & B-C \\
0 & 1
\end{array}\right) \quad \text { or } \quad\left(\begin{array}{cc}
1 & 0 \\
C-B & 1
\end{array}\right)
$$

and

$$
C-B=e^{\eta} \gamma^{*}, \quad \text { or } \quad C-B=e^{-\eta} \gamma^{*},
$$

which are similarity transformations of the triangularr matrices given in equation (1).

Therefore, $A B C D$ is a similarity transformation of one of the Wigner matrices given in equation (11). We thus write

$$
A B C D=S W S^{-1}
$$

where $W$ is the Wigner matrix given in equation (11), and the similarity transformation matrix is

$$
S=\left(\begin{array}{cc}
\cos (\delta / 2) & -\sin (\delta / 2) \\
\sin (\delta / 2) & \cos (\delta / 2)
\end{array}\right)\left(\begin{array}{cc}
e^{\eta / 2} & 0 \\
0 & e^{-\eta / 2}
\end{array}\right)
$$

This is a rotation matrix preceded by a squeeze matrix. 
If there are four different Wigner matrices, calculations become very cumbersome in practical applications. There are no problems if only one Wigner matrix, such as that of equation (23), is applicable throughout the problem as in the case of laser cavities discussed in Sec. 4.

If two or more forms are involved in one problem, as in the case of multilayer optics discussed in Sec. 5, the problem could be uncontrollable. Thus, we are led to consider one mathematical expression for all four Wigner matrices. For this purpose, let us consider the form

$$
\left(\begin{array}{cc}
\cos (\theta / 2) & -\sin (\theta / 2) \\
\sin (\theta / 2) & \cos (\theta / 2)
\end{array}\right)\left(\begin{array}{cc}
\cosh \lambda & \sinh \lambda \\
\sinh \lambda & \cosh \lambda
\end{array}\right)\left(\begin{array}{cc}
\cos (\theta / 2) & -\sin (\theta / 2) \\
\sin (\theta / 2) & \cos (\theta / 2)
\end{array}\right) .
$$

This is a special case of the Bargmann decomposition [14, and its physical interpretation in special relativity is discussed in the literature [15]. In this paper, we consider this form purely for mathematical convenience for discussing periodic systems. After multiplication, the above expression can be compressed into

$$
\left(\begin{array}{cc}
(\cosh \lambda) \cos \theta & -(\cosh \lambda) \sin \theta+\sinh \lambda \\
(\cosh \lambda) \sin \theta+\sinh \lambda & (\cosh \lambda) \cos \theta
\end{array}\right),
$$

with equal diagonal elements. This matrix has two independent parameters. Indeed, this is another form of the $J F G J$ matrix with

$$
\begin{aligned}
& J=(\cosh \lambda) \cos \theta, \\
& F=-(\cosh \lambda) \sin \theta+\sinh \lambda, \\
& G=(\cosh \lambda) \sin \theta+\sinh \lambda .
\end{aligned}
$$

When $\lambda=0$, it becomes

$$
\left(\begin{array}{cc}
\cos \theta & -\sin \theta \\
\sin \theta & \cos \theta
\end{array}\right)
$$

while it takes the form

$$
\left(\begin{array}{cc}
\cosh \lambda & \sinh \lambda \\
\sinh \lambda & \cosh \lambda
\end{array}\right)
$$

when $\theta=0$.

When $\tanh \lambda= \pm \sin \theta$, the equi-diagonal matrix of equation (31) becomes

$$
\left(\begin{array}{cc}
1 & 0 \\
2 \sinh \eta & 1
\end{array}\right), \quad\left(\begin{array}{cc}
1 & -2 \sinh \eta \\
0 & 1
\end{array}\right),
$$

respectively. 


\section{Laser Cavities}

The laser cavity consists of two concave mirrors separated by distance $s$. The mirror matrix takes the form

$$
\left(\begin{array}{cc}
1 & 0 \\
-2 / R & 1
\end{array}\right)
$$

where $R$ is the radius of the concave mirror. The separation matrix is

$$
\left(\begin{array}{ll}
1 & s \\
0 & 1
\end{array}\right)
$$

If we start the cycle from one of the two mirrors one complete cycle consists of

$$
\left(\begin{array}{cc}
1 & 0 \\
-2 / R & 1
\end{array}\right)\left(\begin{array}{ll}
1 & s \\
0 & 1
\end{array}\right)\left(\begin{array}{cc}
1 & 0 \\
-2 / R & 1
\end{array}\right)\left(\begin{array}{ll}
1 & s \\
0 & 1
\end{array}\right) .
$$

If we start the at the position $d$ from the mirror, then the complete cycle becomes

$$
\left(\begin{array}{ll}
1 & d \\
0 & 1
\end{array}\right)\left(\begin{array}{cc}
1 & 0 \\
-2 / R & 1
\end{array}\right)\left(\begin{array}{ll}
1 & s \\
0 & 1
\end{array}\right)\left(\begin{array}{cc}
1 & 0 \\
-2 / R & 1
\end{array}\right)\left(\begin{array}{cc}
1 & s-d \\
0 & 1
\end{array}\right)
$$

since

$$
\left(\begin{array}{ll}
1 & s \\
0 & 1
\end{array}\right)=\left(\begin{array}{cc}
1 & s-d \\
0 & 1
\end{array}\right)\left(\begin{array}{ll}
1 & d \\
0 & 1
\end{array}\right)
$$

Thus, one complete cycle consists of two repeated applications of the half cycle, which can be written as

$$
L=\left(\begin{array}{ll}
1 & d \\
0 & 1
\end{array}\right)\left(\begin{array}{cc}
1 & 0 \\
-2 / R & 1
\end{array}\right)\left(\begin{array}{cc}
1 & s-d \\
0 & 1
\end{array}\right)
$$

which becomes

$$
L=\left(\begin{array}{cc}
1-2 d / R & (1-2 d / R)(s-d)+d \\
-2 / R & 1-2(s-d) / R
\end{array}\right) .
$$

One complete cycle then becomes $L^{2}$. There is however inconvenient feature of this expression is that the off-diagonal elements have different dimensions, while the diagonal elements are dimensionless. In order to deal with this problem, we write this expression as a similarity transformation

$$
\left(\begin{array}{cc}
\sqrt{s} & 0 \\
0 & 1 / \sqrt{s}
\end{array}\right)\left(\begin{array}{cc}
1-2 d / R & (1-2 d / R)(s-d) / s+d / s \\
-2 s / R & 1-2(s-d) / R
\end{array}\right)\left(\begin{array}{cc}
1 / \sqrt{s} & 0 \\
0 & \sqrt{s}
\end{array}\right) .
$$


The elements of the middle matrix are now dimensionless. Let us now use the notations $a=d / s$ and $b=2 / R$. Then we can work with the normalized $L$, which takes the form

$$
L=\left(\begin{array}{cc}
1-a b & 1-a b(1-a) \\
-b & 1-b(1-a)
\end{array}\right)
$$

where $s=1$, according to the normalization defined in equation (43). We can now bring this form into a equi-diagonal matrix. We can write this expression in the form of equation (19) with

$$
\begin{aligned}
& \tan \delta=\frac{b-2 a b}{1-b-a b(1-a)}, \\
& F=\frac{1}{2}\left((1+b)-a b(1-a)+\sqrt{(b-2 a b)^{2}+\left[1-b\left(1+a-a^{2}\right)\right]^{2}}\right) \\
& G=\frac{1}{2}\left(a b(1-a)-(1+b)+\sqrt{(b-2 a b)^{2}+\left[1-b\left(1+a-a^{2}\right)\right]^{2}}\right) .
\end{aligned}
$$

We can now write the $A B C D$ matrix for one complete cycle as a similarity transformation

$$
\left[S R\left(2 \theta^{*}\right) S^{-1}\right]^{2}=S R\left(4 \theta^{*}\right) S^{-1}
$$

and

$$
R\left(2 \theta^{*}\right)=\left(\begin{array}{cc}
\cos \theta^{*} & -\sin \theta^{*} \\
\sin \theta^{*} & \cos \theta^{*}
\end{array}\right)
$$

with

$$
\cos \theta^{*}=1-\frac{b}{2}
$$

The similarity transformation matrix $S$ is in equation (29) and takes the form

$$
S=\left(\begin{array}{cc}
e^{\eta / 2} \cos (\delta / 2) & -e^{-\eta / 2} \sin (\delta / 2) \\
e^{\eta / 2} \sin (\delta / 2) & e^{-\eta / 2} \cos (\delta / 2)
\end{array}\right)
$$

with

$$
e^{2 \eta}=-\frac{F}{G}
$$

where $F, G$, and $\delta$ are given in equation (45).

Thus, for $N$ cycles, the $A B C D$ matrix becomes

$$
\left[S R\left(2 \theta^{*}\right) S^{-1}\right]^{2 N}=S R\left(4 N \theta^{*}\right) S^{-1}
$$


where we have used the logarithmic property of the Wigner matrix.

When the cycle starts from the midpoint in the cavity, $a=1 / 2$, and the half-cycle matrix becomes

$$
L=\left(\begin{array}{cc}
1-b / 2 & 1-b / 4 \\
-b & 1-b / 2
\end{array}\right)
$$

and $\delta$ becomes zero. We do not need the rotation matrix, and this matrix can be written as

$$
L=\left(\begin{array}{cc}
\cos \theta^{*} & e^{\eta} \sin \theta^{*} \\
-e^{-\eta} \sin \theta^{*} & \cos \theta^{*}
\end{array}\right)
$$

with

$$
\cos \theta^{*}=1-\frac{b}{2}, \quad e^{2 \eta}=\frac{4-b}{4 b} .
$$

The matrix $L$ can of course be written as a similarity transformation

$$
L=\left(\begin{array}{cc}
e^{\eta / 2} & 0 \\
0 & e^{-\eta / 2}
\end{array}\right)\left(\begin{array}{cc}
\cos \theta^{*} & \sin \theta^{*} \\
-\sin \theta^{*} & \cos \theta^{*}
\end{array}\right)\left(\begin{array}{cc}
e^{-\eta / 2} & 0 \\
0 & e^{\eta / 2}
\end{array}\right) .
$$

For the laser consisting of $N$ cycles, the $A B C D$ matrix becomes

$$
L^{2 N}=\left(\begin{array}{cc}
e^{\eta / 2} & 0 \\
0 & -e^{-\eta / 2}
\end{array}\right)\left(\begin{array}{cc}
\cos \left(N \theta^{*}\right) & \sin \left(N \theta^{*}\right) \\
-\sin \left(N \theta^{*}\right) & \cos \left(N \theta^{*}\right)
\end{array}\right)\left(\begin{array}{cc}
e^{-\eta / 2} & 0 \\
0 & -e^{\eta / 2}
\end{array}\right),
$$

and thus

$$
L^{2 N}=\left(\begin{array}{cc}
\cos \left(N \theta^{*}\right) & e^{\eta} \sin \left(N \theta^{*}\right) \\
-e^{-\eta} \sin \left(N \theta^{*}\right) & \cos \left(N \theta^{*}\right)
\end{array}\right) .
$$

This is the result we obtained in our previous paper on laser cavities [6]. The point of this paper is we can start the cycle from an arbitrary point, by introducing the parameter $\delta$.

When the cycle begins from one of the lenses, $a=0$, and the $L$ matrix becomes

$$
L=\left(\begin{array}{cc}
1 & 1 \\
-b & 1-b
\end{array}\right) .
$$

This expression can first be brought to the equi-diagonal form by a rotation matrix with the rotation angle

$$
\tan \delta=\left(\frac{b}{1-b}\right)
$$


according to equation (20). The equi-diagonal matrix then takes the form of equation (53) or equation (54) with

$$
\begin{aligned}
& \cos \theta^{*}=1-\frac{b}{2} \\
& e^{2 \eta}=\frac{(1+b)+\sqrt{b^{2}+(1-b)^{2}}}{(1+b)-\sqrt{b^{2}+(1-b)^{2}}} .
\end{aligned}
$$

Indeed the $L$ matrix of equation (57) is a similarity transformation of the Wigner matrix

$$
\left(\begin{array}{cc}
\cos \theta^{*} & \sin \theta^{*} \\
-\sin \theta^{*} & \cos \theta^{*}
\end{array}\right)
$$

with the transformation matrix of the form given in equation (29), which is a rotation preceded by a squeeze. Then it is straight-forward to calculate the $A B C D$ matrix for $N$ cycles.

\section{$5 \quad$ Multilayer Optics}

In multilayer optics, we are led to consider two beams moving in opposite directions [1]. One is the incident beam and the other is the reflected beam. We can represent them as a two component column matrix

$$
\left(\begin{array}{c}
E_{+} e^{i k x} \\
E_{-} e^{-i k x}
\end{array}\right)
$$

where the upper and lower components correspond to the incoming and reflected beams respectively. For a given frequency, the wave number depends on the index of the refraction. Thus, if the beam travels along the distance d, the column matrix should be multiplied by the two-by-two matrix [1]

$$
\left(\begin{array}{cc}
e^{i \alpha / 2} & 0 \\
0 & e^{-i \alpha / 2}
\end{array}\right)
$$

where $\alpha / 2=k d$. Thus, the propagation matrices for two different media can be represented by

$$
\left(\begin{array}{cc}
e^{i \alpha_{1} / 2} & 0 \\
0 & e^{-i \alpha_{1} / 2}
\end{array}\right), \quad\left(\begin{array}{cc}
e^{i \alpha_{2} / 2} & 0 \\
0 & e^{-i \alpha_{2} / 2}
\end{array}\right)
$$


respectively, with $\alpha_{1} / 2=k_{1} d$ and $\alpha_{2} / 2=k_{2} d$.

If the beam propagates along the first medium and meets the boundary at the second medium, it will be partially reflected and partially transmitted. The boundary matrix is [1, 4]

$$
\left(\begin{array}{cc}
\cosh (\mu / 2) & \sinh (\mu / 2) \\
\sinh (\mu / 2) & \cosh (\mu / 2)
\end{array}\right)
$$

with

$$
\cosh (\mu / 2)=1 / t_{12}, \quad \sinh (\mu / 2)=r_{12} / t_{12},
$$

where $t_{12}$ and $r_{12}$ are the transmission and reflection coefficients respectively, and they satisfy $\left(r_{12}^{2}+t_{12}^{2}\right)=1$. The boundary matrix for the second to first medium is the inverse of the above matrix and can be written as

$$
\left(\begin{array}{cc}
\cosh (\mu / 2) & -\sinh (\mu / 2) \\
-\sinh (\mu / 2) & \cosh (\mu / 2)
\end{array}\right)
$$

Thus one complete cycle, starting from the second medium, consists of

$$
\begin{aligned}
& \left(\begin{array}{cc}
\cosh (\mu / 2) & \sinh (\mu / 2) \\
\sinh (\mu / 2) & \cosh (\mu / 2)
\end{array}\right)\left(\begin{array}{cc}
e^{i \alpha_{1} / 2} & 0 \\
0 & e^{-i \alpha_{1} / 2}
\end{array}\right) \\
& \quad \times\left(\begin{array}{cc}
\cosh (\mu / 2) & -\sinh (\mu / 2) \\
-\sinh (\mu / 2) & \cosh (\mu / 2)
\end{array}\right)\left(\begin{array}{cc}
e^{i \alpha_{2} / 2} & 0 \\
0 & e^{-i \alpha_{2} / 2}
\end{array}\right) .
\end{aligned}
$$

This expression can be unitarily transformed to [16]

$$
\begin{aligned}
M= & \left(\begin{array}{cc}
e^{\mu / 2} & 0 \\
0 & e^{-\mu / 2}
\end{array}\right)\left(\begin{array}{cc}
\cos \left(\alpha_{1} / 2\right) & -\sin \left(\alpha_{1} / 2\right) \\
\sin \left(\alpha_{1} / 2\right) & \cos \left(\alpha_{1} / 2\right)
\end{array}\right)\left(\begin{array}{cc}
e^{-\mu / 2} & 0 \\
0 & e^{\mu / 2}
\end{array}\right) \\
& \times\left(\begin{array}{cc}
\cos \left(\alpha_{2} / 2\right) & -\sin \left(\alpha_{2} / 2\right) \\
\sin \left(\alpha_{2} / 2\right) & \cos \left(\alpha_{2} / 2\right)
\end{array}\right) .
\end{aligned}
$$

The first three matrices in the above expression can be compressed to

$$
\left(\begin{array}{cc}
\cos \left(\alpha_{1} / 2\right) & -e^{\mu} \sin \left(\alpha_{1} / 2\right) \\
e^{-\mu} \sin \left(\alpha_{1} / 2\right) & \cos \left(\alpha_{1} / 2\right)
\end{array}\right)
$$

and thus, according to equation (31) and equation (33), to

$$
\left(\begin{array}{cc}
\cos \left(\theta_{1}\right) \cosh \lambda & -\sin \left(\theta_{1}\right) \cosh \lambda+\sinh \lambda \\
\sin \left(\theta_{1}\right) \cosh \lambda+\sinh \lambda & \cos \left(\theta_{1}\right) \cosh \lambda
\end{array}\right)
$$


with

$$
\begin{aligned}
& \cosh \lambda=(\cosh \mu) \sqrt{1-\cos ^{2}\left(\alpha_{1} / 2\right) \tanh ^{2} \mu}, \\
& \cos \theta_{1}=\frac{\cos \left(\alpha_{1} / 2\right)}{(\cosh \mu) \sqrt{1-\cos ^{2}\left(\alpha_{1} / 2\right) \tanh ^{2} \mu}} .
\end{aligned}
$$

Then the matrix $M$ of equation (68) can be written as

$$
\begin{aligned}
M= & \left(\begin{array}{cc}
\cos \left(\theta_{1} / 2\right) & -\sin \left(\theta_{1} / 2\right) \\
\sin \left(\theta_{1} / 2\right) & \cos \left(\theta_{1} / 2\right)
\end{array}\right)\left(\begin{array}{cc}
\cosh \lambda & \sinh \lambda \\
\sinh \lambda & \cosh \lambda
\end{array}\right) \\
& \times\left(\begin{array}{cc}
\cos \left(\theta_{2} / 2\right) & -\sin \left(\theta_{2} / 2\right) \\
\sin \left(\theta_{2} / 2\right) & \cos \left(\theta_{2} / 2\right)
\end{array}\right),
\end{aligned}
$$

with

$$
\theta_{2}=\theta_{1}+\alpha_{2}
$$

We can now write this expression as a similarity transformation of a matrix with equal diagonal elements. Explicitly,

$$
\left(\begin{array}{cc}
\cos (\delta / 2) & -\sin (\delta / 2) \\
\sin (\delta / 2) & \cos (\delta / 2)
\end{array}\right)\left(\begin{array}{cc}
J & F \\
G & J
\end{array}\right)\left(\begin{array}{cc}
\cos (\delta / 2) & \sin (\delta / 2) \\
-\sin (\delta / 2) & \cos (\delta / 2)
\end{array}\right),
$$

where

$$
\begin{aligned}
& \left(\begin{array}{cc}
J & F \\
G & J
\end{array}\right)=\left(\begin{array}{cc}
\cos (\theta / 2) & -\sin (\theta / 2) \\
\sin (\theta / 2) & \cos (\theta / 2)
\end{array}\right)\left(\begin{array}{cc}
\cosh \lambda & \sinh \lambda \\
\sinh \lambda & \cosh \lambda
\end{array}\right) \\
& \times\left(\begin{array}{cc}
\cos (\theta / 2) & -\sin (\theta / 2) \\
\sin (\theta / 2) & \cos (\theta / 2)
\end{array}\right),
\end{aligned}
$$

with

$$
\delta=\frac{1}{2}\left(\theta_{1}-\theta_{2}\right), \quad \theta=\frac{1}{2}\left(\theta_{1}+\theta_{2}\right) .
$$

The above three matrices can be compressed to one equi-diagonal matrix

$$
\left(\begin{array}{cc}
\cosh \lambda \cos \theta & -\cosh \lambda \sin \theta+\sinh \lambda \\
\cosh \lambda \sin \theta+\sinh \lambda & \cosh \lambda \cos \theta
\end{array}\right) .
$$

This matrix can now be written as a similarity transformation of one of the four Wigner matrices. 
When the off-diagonal elements have opposite signs, we can write this as

$$
\left(\begin{array}{cc}
e^{\sigma / 2} & 0 \\
0 & e^{-\sigma / 2}
\end{array}\right)\left(\begin{array}{cc}
\cos \omega & -\sin \omega \\
\sin \omega & \cos \omega
\end{array}\right)\left(\begin{array}{cc}
e^{-\sigma / 2} & 0 \\
0 & e^{\sigma / 2}
\end{array}\right),
$$

with

$$
\begin{aligned}
& \cosh \omega=\cosh \lambda \cos \theta, \\
& e^{2 \sigma}=\frac{\cosh \lambda \sin \theta-\sinh \lambda}{\cosh \lambda \sin \theta+\sinh \lambda} .
\end{aligned}
$$

When the off-diagonal elements have the same sign, we should write

$$
\left(\begin{array}{cc}
J & F \\
G & J
\end{array}\right)=\left(\begin{array}{cc}
e^{\sigma / 2} & 0 \\
0 & e^{-\sigma / 2}
\end{array}\right)\left(\begin{array}{cc}
\cosh \chi & \sinh \chi \\
\sinh \chi & \cosh \chi
\end{array}\right)\left(\begin{array}{cc}
e^{-\sigma / 2} & 0 \\
0 & e^{\sigma / 2}
\end{array}\right),
$$

with

$$
\begin{aligned}
& \cosh \chi=\cosh \lambda \cos \theta, \\
& e^{2 \sigma}=\frac{\sinh \lambda-\cosh \lambda \sin \theta}{\cosh \lambda \sin \theta+\sinh \lambda} .
\end{aligned}
$$

If one of the off-diagonal elements vanishes, the matrix takes the form

$$
\left(\begin{array}{cc}
1 & -2 \sinh \lambda \\
0 & 1
\end{array}\right) \text { or } \quad\left(\begin{array}{cc}
1 & 0 \\
2 \sinh \lambda & 1
\end{array}\right) \text {. }
$$

Now the diagonalization of the above expression is straight-forward according to the mathematical tool we have developed in this paper. So is the computation of

$$
M^{N} \text {. }
$$

The computation of this process was started in reference [5]. There, the cycle had to start from the midway in one of the media, but no explanation was given why. In this paper, by introducing the $R(\delta)$ matrix, we can start from an arbitrary point. Indeed, this is a physical interpretation of this rotation matrix.

When the cycle starts from an arbitrary point in the medium, we can adjust the value of the rotation angle $\delta$ as we did in Sec. 4, 


\section{Concluding Remarks}

In dealing with a two-by-two matrix, we are accustomed to think it can be diagonalized and can be brought to the diagonal matrix by a rotation. This paper shows this assumption is not always true, because the $A B C D$ matrix is not always a rotation matrix.

It has been shown that the $A B C D$ matrix can be brought to one of the four one-parameter Wigner matrices through a similarity transformation, and the similarity transformation is a rotation followed by a squeeze transformation.

These one-parameter Wigner matrices have the logarithmic property which allows us to calculate repeated applications by multiplying the parameter by an integer. This property is transmitted to its similarity transformation, since

$$
\left(S W S^{-1}\right)^{N}=S W^{N} S^{-1} .
$$

This mathematical technique is applied to laser cavities and multilayer systems. However, it is clear from the literature that the mathematical result stated in this paper is a result of our efforts to understand physical systems.

This mathematical instrument is applicable to other periodic systems in physics, such as one-dimensional scattering problems in quantum mechanics [17, 18], especially in condensed-matter physics. We can also broaden our scope to look into applications in space-time symmetries of elementary particles in view of the fact that the Wigner matrices used in this paper are from Wigner's 1939 paper on symmetries in the Lorentz-covariant world [12, 19].

For periodic systems, many authors used different approaches. SanchezSoto and his co-authors used the conformal representation of the $S p(2)$ group to attack the problem [4, 20]. This is possible due to the fact that the transformations of this group can be translated into conformal transformations as noted by Bargmann [14].

Another interesting approach to the periodic system is to use mathematical induction [21. It is possible to assume first that the $A B C D$ matrix is known for $N$ cycles, and then compute the system for $N+1$ cycles. 


\section{References}

[1] Azzam R A M and Bashara I 1977 Ellipsometry and Polarized Light (North-Holland, Amsterdam)

[2] Başkal S and Kim Y S 2007 Phys. Rev. E 63 056606-056611

[3] Başkal S and Kim Y S Phys. Rev. E 67 056601-056608

[4] Monzon J J and Sanchez-Soto L L 2000 J. Opt. Soc. Am. A 17 1475-1481

[5] Georgieva E and Kim Y S 2003 Phys. Rev. E 68 026606-026612

[6] Başkal S and Kim Y S 2002 Phys. Rev. E 66 026604-026609

[7] Yuen H P 1976 Phys. Rev. A 13 2226-2243

[8] Kim Y S and Noz M E 1991 Phase Space Picture of Quantum Mechanics (World Scientific, Singapore) Chap 5

[9] Kim Y S and Noz M E 1983 Am. J. Phys. 51 368-375

[10] Gillemin V and Sternberg S 1984 Symplectic techniques in physics (Cambridge University Press, Cambridge) Chap 1.

[11] Lang S $1985 S L_{2}(R)$ (Springer-Verlag, Heidelberg) Chap 1 and 5

[12] Wigner E 1939 Ann. Math. 40 149-204

[13] Kim Y S and Noz M E 1986 Theory and Applications of the Poincaré Group (Reidel, Dordrecht) Chaps 3, 4 and 9.

[14] Bargmann V 1947 Ann. Math. 48 568-641

[15] Han D and Kim Y S 1988 Phys. Rev. A 37 4494-4496

[16] Georgieva E and Kim Y S 2001 Phys. Rev. E 64 026602-026607

[17] Sprung D W L, Wu Hu, and Martorell J 1993 Am. J. Phys. 61 1118-1124

[18] Griffiths D J and Steinke C A 2001 Am. J. Phys. 69 137-154

[19] Han D, Kim Y S, and Son D 1986 J. Math. Phys. 27 2228-2235 
[20] Sánchez-Soto L L, Cariñena J F, Barriuso A G, and Monzon L L 2005 Eur. J. Phys. 26 469-480, and the references contained in this paper.

[21] Kildemo M, Hunderi O, and Drevillon B, 1997 J. Opt. Soc. Am. A 14 931-939 